\title{
TWO MORE REPRESENTATION PROBLEMS
}

\author{
by ANDREW BREMNER and RICHARD K. GUY* \\ (Received 7th October 1994)
} \begin{abstract}
those which may be represented by $x / y+y / z+z / x$, where $x, y, z$ are integers. For example,

$$
\begin{aligned}
& x=-3888953655693309094309277243253295616000, \\
& y=870614350109377939730940722158565152629, \\
& z=-211788680591112853611774198484237121509
\end{aligned}
$$

satisfy $(x+y+z)^{3} /(x y z)=-47$, and

$$
\begin{aligned}
& x=10695607136243980529530429582617991136107507407713992824, \\
& y=-123256655541019041417443728014061733054947136623569984, \\
& z=8446121230200308492574953446465639841507828834632079329
\end{aligned}
$$
\end{abstract}

We discuss the problem of finding those integers which may be represented by $(x+y+z)^{3} / x y z$, and also

satisfy $x / y+y / z+z / x=-86$.

1991 Mathematics subject classification: 11D25, $11 \mathrm{G} 05$.

\section{Introduction}

In [2] we discussed Melvyn Knight's problem of finding those integers representable in the form

$$
n=(x+y+z)\left(\frac{1}{x}+\frac{1}{y}+\frac{1}{z}\right)
$$

and exhibited triples of integers $(x, y, z)$ for all but 99 of the possible $n$ in the range $-1000 \leq n \leq 1000$. Analogous problems have arisen elsewhere, and in [8] it was asked to find all integers representable by

$$
\frac{(x+y+z)^{2}}{x y z}=n,
$$

but it turns out that only a small finite number of positive $n$ are so representable, while

* Supported by Nat. Sci. Engg. Res. Council of Canada grant A-4011. 
negative integers are given by $(x, y, z)=\left(n,-n, n^{3}\right)$. Homogeneity is desirable (because of the underlying elliptic curve structure), and a better question [9] is to ask for representations

$$
\frac{(x+y+z)^{3}}{x y z}=n
$$

A representation problem that is closely related to (1) has attracted some historical interest:

$$
\frac{x}{y}+\frac{y}{z}+\frac{z}{x}=n
$$

Cassels [3] and Cassels and Sansone [4] show there are no integer solutions for (2) when $n=1$; see also Sierpiński [13]. The equation merits attention in Sierpiński [14], who asks for all solutions in the cases $n=1,2,3$. From Konhauser, Velleman and Wagon [11], one learns that Underwood Dudley had given the solutions $(9,162,4)$, $(72,162,4)$ and $(350,196,5)$, each for $n=41$. Peter Montgomery observes that if $x, y, z$ have no factor in common, then (2) implies that there exist integers $a, b, c$ such that $x=a^{2} b, y=b^{2} c, z=c^{2} a$. Then

$$
n=\frac{x}{y}+\frac{y}{z}+\frac{z}{x}=\frac{a^{2}}{b c}+\frac{b^{2}}{c a}+\frac{c^{2}}{a b}=\frac{a^{3}+b^{3}+c^{3}}{a b c} .
$$

In the form (3), the representation problem at (2) has been studied by Dofs [5, 6], who gives some parametric solutions and a table of numerical solutions for some $n$ in the range $-81 \leq n \leq 80$. See also Craig [5], Mohanty [12], and Thomas and Vasquez [15]. Notice that $\left(a^{3}, b^{3}, c^{3}, n^{3}\right)$ at (3) is now a solution to the first representation problem (1). In the reverse direction, a solution of (1) in which $n$ is a perfect cube gives rise to a point on the underlying elliptic curve which (assuming it is not a torsion point) may be tripled to give a point corresponding to $x, y, z$ being all cubes, and hence giving rise to a solution of (2) for $n^{1 / 3}$.

An initial computer search was carried out for solutions to (1) and (3) by Peter Montgomery. For (1), the search was over the range $1 \leq x \leq y \leq z \leq 46,300$, and 539 solutions were found, involving 501 different values of $n, 69$ of them in the interval $27 \leq n \leq 1000$. For (3), the search range was $\max (|a|,|b|,|c|) \leq 2000,|n|<10^{6}$, with 800 solutions found.

A descent can be carried out on (3) by means of Sylvester's transformations, given by Dofs [6].

Let $U=n a+3 b+3 c, V=3 a+n b+3 c, W=3 a+3 b+n c$

with inverse

$$
a: b: c=(n+3) U-3 V-3 W:-3 U+(n+3) V-3 W:-3 U-3 V+(n+3) W
$$


Then (3) becomes

$$
\left(n^{2}+3 n+9\right)(U+V+W)^{3}=(n+6)^{3} U V W
$$

so that there exist integers $P, Q, R$ and $\alpha, \beta, \gamma$ with $\alpha \beta \gamma=n^{2}+3 n+9$, satisfying

$$
\alpha P^{3}+\beta Q^{3}+\gamma R^{3}=(n+6) P Q R .
$$

One can now search (4) for solutions, and we made several additions in this manner to our list of solutions of (2). However, the main thrust of this study is to investigate in each of the problems the underlying family of elliptic curves. Rene Schoof used the family (3) in a lecture at Bordeaux in May 1995, to illustrate the ideas of 3-descent. He computed many of the smaller solutions, and one or two others by the calculation of Heegner points. His tables were carried further by Cremona (using a customized 2-descent) and by Fermigier (by direct computation), leaving gaps at $n=-86,-80,-63,72,73,92,99$.

We extend our grateful thanks to Peter Montgomery, both for discussion of the problems and for his solution search to the pair of equations, and to John Cremona, Erik Dofs, and René Schoof, who all commented fruitfully on the first draft of this paper.

\section{Two families of elliptic curves}

Solutions to (1) are obtained by writing

$$
\frac{x, y}{z}=\frac{ \pm Y-n X-4}{8}
$$

so that they correspond to rational points on the elliptic curves

$$
Y^{2}=n^{2} X^{3}+(n X+4)^{2}
$$

the inverse transformation being

$$
(X, Y)=\left(\frac{-4(x+y+z)}{n z}, \frac{4(x-y)}{z}\right)
$$

Similarly, solutions to (2) may be found by putting

$$
x: y: z=-X^{2}: 2(n X+4-Y): 4 X
$$

yielding the family

$$
Y^{2}=X^{3}+(n X+4)^{2}
$$


whose rational points are

$$
(X, Y)=\left(\frac{-4 x}{z}, \frac{4\left(2 x y+z^{2}-n z x\right)}{z^{2}}\right)
$$

In terms of $a, b, c$ at (3), then

$$
(X, Y)=\left(-\frac{4 a b}{c^{2}}, \frac{4\left(b^{3}-a^{3}\right)}{c^{3}}\right)
$$

The discriminants of (6) and (9) are $2^{12} n^{8}(n-27)$ and $2^{12}\left(n^{3}-27\right)$, respectively, so that the former is singular just when $n=0$ or 27 , and the latter only when $n=3$. All members of both families have points of inflexion $(0, \pm 4)$ of order 3 , and the torsion group is, in general, $\mathbb{Z} / 3 \mathbb{Z}$. Exceptionally, the cubic may have a rational root, giving a point of order 2 and a torsion group $\mathbb{Z} / 6 \mathbb{Z}$. For the first family this occurs just for $n=-1,2,(27)$, 32, 54 and 125 and for the second family when $n=-1,(3)$ and 5. These statements about the torsion group have been independently verified by the students of Nigel Boston's 'Elliptic Curves by Computer' class at the University of Illinois, UrbanaChampaign. One of these students, Sharon Kineke, makes the following interesting observation [10]: construct four binary trees from the root triples $(1,1,1),(1,1,2)$, $(1,2,3)$ and $(1,4,5)$ such that the triple $(x, y, z)$ gives rise to the triples $\left(x, z,(x+z)^{2} / y\right)$ and $\left(y, z,(y+z)^{2} / x\right)$. Each node $(\mathcal{X}, \mathcal{Y}, \mathcal{Z})$ of these trees makes $(\mathcal{X}+\mathcal{Y}+\mathcal{Z})^{2} / \mathcal{X Y} \mathcal{Z}$ an integer (and, a fortiori, $(\mathcal{X}+\mathcal{Y}+\mathcal{Z})^{3} / \mathcal{X Y \mathcal { Z }}$ an integer). This provides an infinite set of solutions to the representation problem $(x+y+z)^{2} / x y z=n$ of $[8]$ for the respective cases $n=9,8,6$ and 5, and, by appropriate multiples, for their divisors $n=1,2,3$ and 4 . The first tree includes the triples $\left(1, f_{2 n-1}^{2}, f_{2 n+1}^{2}\right)$ where $f_{j}$ is the $j$-th Fibonacci number, so these are solutions to $(x+y+z)^{2} / x y z=9$, and show that $27 f_{2 n-1} f_{2 n+1}$ can always be represented by $(x+y+z)^{3} / x y z$.

Solutions to each of our representation problems occur in orbits of six. In the first, these are simply the permutations of $x, y, z$; for the second, cyclic permutations of $(x, y, z)$ and of their reciprocals in reverse order, $(1 / z, 1 / y, 1 / x)$. Note that in either instance it suffices to find rational values of $(x, y, z)$; integer solutions are retrieved by multiplying by a suitable factor. In each problem integer solutions may be normalized so that $\operatorname{gcd}(x, y, z)=1$. In the first problem it may further be assumed that $x, y, z$ are coprime in pairs.

In the first problem the solutions $(x, y, z ; n)=(1,1,-1 ;-1),(1,1,-4 ; 2)$, $(1,1,2 ; 32),(1,1,4 ; 54),(1,1,8 ; 125)$ come from torsion points and are essentially unique. For $n=27$, the solution $(1,1,1)$ is essentially unique as a positive representation, but

$$
\left((m-1)^{3},-(m+1)^{3}, 8\right)
$$

also serves for any rational $m$, e.g., $(1,1,-8),(1,-27,8),(27,-125,8)$. 
In the second problem, $n=3$ is given by $(1,1,1)$ or by

$$
\left(2(m-1)^{2},-4(m+1),(m-1)(m+1)^{2}\right)
$$

with $m$ rational, the reciprocal solutions being given by changing the sign of $m$ and the cyclic order:

$$
\left(2(m+1)^{2},-(m+1)(m-1)^{2}, 4(m-1)\right) .
$$

There is a 2-isogeny between the curves for $n=-1$ and $n=5$. They have rank 0 , and the solutions $(1,1,-1 ;-1)$ and $(1,2,4 ; 5)$ come from the respective torsion points of order six, namely, $(4,-8)$ and $(-8,-28)$.

In the first problem, the curves consist of a single branch if $0 \neq n<27$, and of a branch and an 'egg' if $n>27$. Correspondingly in the second problem, the type of curve is determined by $n<3$ or $n>3$. From (5) and (8) we see that in both problems the necessary and sufficient conditions for a representation of $n$ by positive $x, y, z$ are

$$
n>0 \text { and } X<-4 / n
$$

Thus there are positive representations precisely when the curve contains an 'egg' component possessing a rational point; just as in Melvyn Knight's problem [2].

\section{3. $L$-series calculations}

In order to determine where it would be profitable to search for solutions, the parity of the rank of each elliptic curve was determined (modulo the Selmer conjecture) by computing the sign of the functional equation for the appropriate $L$-series $L_{E}(s)$. When the sign is +1 , then evaluate $L_{E}(1)$. If nonzero, the rank is zero. If $L_{E}(1)=0$, then the rank is at least 2; now evaluate $L_{E}^{\prime \prime}(1)$. If nonzero, as is the case over the range we considered, then the rank is 2, and the formulae of Gross \& Zagier allow an estimate to be computed of the height regulator of a pair of generators, multiplied by the order of the Shafarevich-Tate group.

When the sign is -1 , then evaluate $L_{E}^{\prime}(1)$. If nonzero, as is the case over the range we considered, then the rank is 1 , and again we can compute an estimate for the height of a generator multiplied by the order of the Shafarevich-Tate group. The initial range which we decided to search for generators of the Mordell-Weil group was $-200 \leq n \leq 200$, but this had to be shortened to $-100 \leq n \leq 100$. Comparison with the tables in Bremner, Guy and Nowakowski [2] is instructive, since the latter are far more extensive. The difference arises from the fact that the elliptic curves at (6) and (9) do not possess rational 2-torsion. It was the possession of a rational 2-division point that enabled the descent arguments of [2] to be carried out in an algorithmic method which could be machine programmed. Here, in order to find the larger entries in Tables 1 and 2, it was 
necessary to treat each case individually as we were unable to construct a generic descent method. The initial computer searches are responsible for entries in the tables up to approximate height 10 to 12 ; and descent arguments, one of which is sketched in section 5 , were needed to fill in the gaps.

Comparison of Table 2 with the table of Dofs [6] shows omissions from the latter of solutions for $n=64$ (which is stated to have no solution), 70,72,73 and $n=-32$, $-48,-50,-53,-56,-63,-65,-67,-80$. Further, $n=76,77$ correspond to rank two examples. However, several of these omissions are supplied in the author's recent paper, Dofs [7].

\section{Parametric solutions}

There are several parametric solutions to the first problem.

If $n=-m^{2}$, then permutations of $(x, y, z)=(1,-1, m)$.

If $n=m^{2}(m+1)$, then permutations of $(x, y, z)=\left(1, m,-(m+1)^{2}\right)$.

If $n=-(m+1)(m-2)^{3}$, then permutations of $(x, y, z)=\left(1,-(m+1)^{2}, m^{3}\right)$.

If $n=-m^{2}(m+1)(m+2)$, then permutations of $(x, y, z)=\left(1,-(m+1)^{2}, m(m+2)^{2}\right)$.

If $n=\left(m^{2}-m+1\right)(m-2)^{3}$, then permutations of $(x, y, z)=\left(1, m^{3},-\left(m^{2}-m+1\right)^{2}\right)$.

For the second problem, numerous parametric solutions are known, see Dofs [6], Mohanty [12], and Thomas and Vasquez [15]. Dofs [7] shows how to produce infinitely many parametrizations, satisfying a recurrence relation.

\section{A descent example}

Although descent arguments are well known in principle, most that occur in the literature correspond to a curve with a rational 2-division point. We feel that it is instructive to give an example where it is necessary to work over algebraic number fields, illustrating some of the techniques that must be used.

We find by descent a point on the curve

$$
y^{2}=x^{3}+(73 x+4)^{2}
$$

Replacing $x, y$ by $x / z^{2}, y / z^{3}$, we treat this equation in the form

$$
y^{2}=x^{3}+\left(73 x z^{2}+4 z^{3}\right)^{2}, \quad(x, z)=1,
$$

so that

$$
\operatorname{Norm}\left(x-\theta z^{2}\right)=y^{2}
$$

where

$$
\theta^{3}+(73 \theta+4)^{2}=0
$$


Using Pari-GP [1] we discover the following facts about the number field $K=\mathbb{Q}(\theta)$.

$K=\mathbb{Q}(\alpha)$, where $\alpha^{3}-73 \alpha-2=0$ and $\theta=-5329-2 \alpha+73 \alpha^{2}$; the ring of integers $\mathcal{O}_{K}$ in $K$ is $\mathbb{Z}[\alpha]$, the ideal class-group is trivial, and a pair of fundamental units is

$\epsilon_{1}=-255+81 \alpha+13 \alpha^{2}$

$\epsilon_{2}=2690940741277869467+98533784900060106769 \alpha+11514090849474001891 \alpha^{2}$

(norm $\epsilon_{1}=+1$, norm $\epsilon_{2}=-1$ ).

From (12) it follows that there are integers $f, g, h$ with

$$
x-\theta z^{2}=(\text { unit })\left(f+g \alpha+h \alpha^{2}\right)^{2}, \quad \text { norm (unit) }=+1,
$$

and the unit can be taken without loss of generality as $1, \epsilon_{1},-\epsilon_{2}$, or $-\epsilon_{1} \epsilon_{2}$. The first choice of unit leads to an infinite descent argument; the second and third choices lead to 2-adically insolvable equations. With unit $=-\epsilon_{1} \epsilon_{2}$, and direct expansion of (13), there result on equating coefficients of $\alpha, \alpha^{2}$ quadratic equations with large coefficients which are unwieldy to treat. Instead, we resort to the following subterfuge: in $D_{K}$ factor the ideals $(2)=\mathfrak{p}_{2}^{2} \mathfrak{p}_{2}^{\prime}$ and $(5)=\mathfrak{p}_{5}^{2} \mathfrak{p}_{5}^{\prime}$ and write $\mathfrak{p}_{2}^{\prime}=\left(q_{2}^{\prime}\right)=(\alpha)$,

$$
p_{5}=\left(q_{5}\right)=\left(29296065+1072729742 \alpha+125353022 \alpha^{2}\right) \text {. }
$$

Calculation then gives $q_{2}^{\prime 2} q_{5}^{2}\left(-\epsilon_{1}^{-1} \epsilon_{2}^{-1}\right)=5036+2 \alpha-69 \alpha^{2}$. Thus, from (13),

$$
\left(5036+2 \alpha-69 \alpha^{2}\right)\left(x-\theta z^{2}\right)=A^{2}, \quad A \equiv 0 \bmod p_{2}^{\prime} p_{5} .
$$

$A$ is accordingly of the form $A=(4 u+2 v)+(-u+2 v+w) \alpha+w \alpha^{2}$, and expanding the above followed by equating coefficients of $\alpha, \alpha^{2}$ gives

$$
\begin{aligned}
4029 u^{2}-34862 u v-152827 u w+73748 v^{2}+635508 v w+1350240 w^{2} & =0 \\
-55 u^{2}+476 u v+2087 u w-1007 v^{2}-8678 v w-18437 w^{2} & =z^{2} .
\end{aligned}
$$

Under the unimodular transformation

$$
\left(\begin{array}{l}
u \\
v \\
w
\end{array}\right)=\left(\begin{array}{rrr}
-3049 & -9744 & 2890 \\
-1634 & -5220 & 1549 \\
212 & 677 & -201
\end{array}\right)\left(\begin{array}{l}
U \\
V \\
W
\end{array}\right),
$$

(14) becomes

$$
\begin{gathered}
-3 U^{2}-U V-U W-2 V W+6 W^{2}=0 \\
9 U^{2}+57 U V+91 V^{2}-17 U W-54 V W+8 W^{2}=z^{2} .
\end{gathered}
$$

The first is parametrized by

$$
U: V: W=s^{2}+2 s t:-3 s^{2}-s t+6 t^{2}: s t+2 t^{2},
$$

and substitution in the second gives

$$
657 s^{4}+328 s^{3} t-2603 s^{2} t^{2}-660 s t^{3}+2660 t^{4}=\text { square, }
$$


and under

$$
\begin{gathered}
\left(\begin{array}{l}
s \\
t
\end{array}\right)=\left(\begin{array}{ll}
13 & -4 \\
10 & -3
\end{array}\right)\left(\begin{array}{l}
S \\
T
\end{array}\right) \\
37 S^{4}-932 S^{3} T+6157 S^{2} T^{2}-3484 S T^{3}+516 T^{4}=\text { square. }
\end{gathered}
$$

Now define $\phi$ by $\phi^{4}-932 \phi^{3}+37.6157 \phi^{2}-37^{2} 3484 \phi+37^{3} 516=0$, so that (15) becomes

$$
\operatorname{Norm}(37 S-\phi T)=37^{3}(\text { square })
$$

PARI informs us that $K=\mathbb{Q}(\phi)=\mathbb{Q}(\beta)$, where $\beta^{4}-2 \beta^{3}-46 \beta^{2}-146 \beta+1673=0$ and $\phi=\frac{1913}{2}+92 \beta-\frac{19}{2} \beta^{2}-3 \beta^{3}$. The ring of integers is $\mathbb{Z}\left[1, \beta, \frac{1}{2}\left(1+\beta^{2}\right), \frac{1}{4}(1+\beta)\left(1+\beta^{2}\right)\right]$, the class-group is trivial, and there is one fundamental unit $\eta$, where $2 \eta=$

75580819871834997764954569092045859117373866656250251392514670806060859765

$+8145350962769745889531810354098633900781417131412831384198747977897517131 \beta$

$-8574132162770733686430399921313822409424090978406737115591614207874149031 \beta^{2}$ $+854009362121281550166075994803607488276541755336529852261557534405204781 \beta^{3}$

In $K$ we factor the ideals $(2)=\mathfrak{P}_{2}^{2} \mathfrak{P}_{2}^{\prime 2},(3)=\mathfrak{P}_{3} \mathfrak{P}_{3}^{\prime}$ and $(37)=\mathfrak{P}_{37} \mathfrak{P}_{37}^{\prime} \mathfrak{P}_{37}^{\prime \prime} \mathfrak{P}_{37}^{\prime \prime \prime}$ with $\phi \equiv \bmod \mathfrak{P}_{2}^{2} \mathfrak{P}_{37}^{\prime} \mathfrak{P}_{37}^{\prime \prime} \mathfrak{P}_{37}^{\prime \prime}$. It follows from (16) that

$$
(37 S-\phi T)=\mathfrak{P}_{37}^{\prime} \mathfrak{P}_{37}^{\prime \prime} \mathfrak{P}_{37}^{\prime \prime \prime} \mathfrak{P} \mathfrak{Y}^{2}
$$

for some integral ideal $\mathfrak{A}$, where $\mathfrak{P}$ is one of the ideals $(1), \mathfrak{P}_{37}^{\prime} \mathfrak{P}_{37}^{\prime \prime}, \mathfrak{P}_{37}^{\prime \prime} \mathfrak{P}_{37}^{\prime \prime \prime}, \mathfrak{P}_{37}^{\prime \prime \prime} \mathfrak{P}_{37}^{\prime}$.

We investigated only $\mathfrak{P}=(1)$ using the identity

$$
q_{2}^{2} q_{3}^{2} q_{37}^{\prime} q_{37}^{\prime \prime} q_{37}^{\prime \prime \prime} \eta=\frac{-3578611}{2}-133604 \beta+\frac{55133}{2} \beta^{2}+6604 \beta^{3}
$$

(where $\mathfrak{P}_{2}=\left(q_{2}\right)$, etc.).

Then (17) becomes

$$
\left(-\frac{3578611}{2}-133604 \beta+\frac{55133}{2} \beta^{2}+6604 \beta^{3}\right)(37 S-\phi T)=A^{2},
$$

$A \equiv \bmod \mathfrak{P}_{2} \mathfrak{P}_{3} \mathfrak{P}_{37}^{\prime} \mathfrak{P}_{37}^{\prime \prime} \mathfrak{P}_{37}^{\prime \prime \prime}$. Accordingly, $A$ can be taken in the form

$$
\begin{aligned}
A= & (31 u+37 v-18 w)+\beta(-13 u+37 v+72 w)+\left(\frac{1+\beta^{2}}{2}\right)(u+3 w) \\
& +\frac{(1+\beta)\left(1+\beta^{2}\right)}{4}(18 u+17 w+37 y)
\end{aligned}
$$


Expanding (18) and equating coefficients of $1, \beta, \beta^{2}, \beta^{3}$ results in quadratic forms which under the transformation

$$
\left(\begin{array}{l}
u \\
v \\
w \\
y
\end{array}\right)=\left(\begin{array}{rrrr}
195 & -140 & -253 & -74 \\
1500 & 290 & -1594 & -359 \\
-660 & -179 & 688 & 150 \\
179 & 144 & -162 & -26
\end{array}\right)\left(\begin{array}{c}
U \\
V \\
W \\
Y
\end{array}\right)
$$

imply

$$
\begin{gathered}
7 U^{2}+6 U V+24 V^{2}+4 U W+2 V W-Y^{2}=0 \\
305 U^{2}+318 U V-192 V^{2}+107 U W+55 V W+15 W^{2}+210 U Y+90 V Y+42 W Y+37 Y^{2}=0
\end{gathered}
$$

At this stage a computer search produces the solution

$$
(U, V, W, Y)=(-815,-645,2772,2275),
$$

which in turn can be traced back to the point $(x, y)$ on the original curve, where

$$
\begin{gathered}
x=-1016423305745800678508556328968 / 1936346342378584154797319700289, \\
y=92466158602758489876800256772058174984104828292 / \\
2694477486671622761439673888324026754597645087 .
\end{gathered}
$$

\section{Tables}

The first table gives solutions to the representation problem at (1). Solutions are listed as pairs $\left\{\left(c_{0}, c_{1}, c_{2}\right)(u, v, w)\right\}$ where $n=c_{0} c_{1} c_{2}$ and $x=c_{0}^{2} u^{3}, y=c_{1}^{2} v^{3}, z=c_{2}^{2} w^{3}$. An asterisk in the first column denotes a singular curve. The second column provides the height of the solution in case the rank is 1 , and the height regulator in case the rank is 2. In all instances the solutions found generate the group of rational points. The third column lists the solutions, the number of such being equal to the rank.

The second table gives solutions $(a, b, c)$ to the representation problem at (3). An asterisk in the first column denotes a singular curve. The second column provides as above the height regulator of the solutions listed in the third column, whose number equals the rank.

The third and fourth tables list a pair of independent solutions for the respective representation problems, in each case in the range $100 \leq|n| \leq 200$ for which the rank is 2 . (The first problem resulted in 181 curves of rank 0, 199 of rank 1 , and 19 of rank 2 , in the range $1 \leq|n| \leq 200$; the second problem in 218 curves of rank 0,139 of rank 1 , and 42 of rank 2 , over the same range).

From the remarks at the end of Section 2, positive representation of $n$ at (1) and (3) occurs precisely when a generator for the group of rational points lies on the 'egg', that is, a generator itself provides a positive representation. Correspondingly, $(u, v, w)$ 
in Tables 1, 3 and $(a, b, c)$ in Tables 2, 4 must all be positive. Notice, for example, from Table 4 that at $n=136$, although the rank equals 2 , neither generator lies on the egg; consequently there are no rational points on the egg, and there is no positive representation of $n=136$ at (3).

Finally in Table 5 we give the instances where the curves (1) and (3) in the range $100<|n| \leq 200$ have rank equal to 1 . More precisely, we list those $n$ for which the sign of the functional equation is -1 and where the first derivative of the $L$-series does not vanish at 1 . By results of Kolyvagin, the rank is equal to 1 provided that the curves are modular. This is implied by the Taniyama-Weil conjecture, and in any specific instance is a finite computation to check. Recent results of Wiles [15] prove the Taniyama-Weil conjecture for semi-stable elliptic curves. René Schoof points out that the curves (3) are semi-stable away from the prime 3 , so that the curves (3) are indeed modular when $3 \not h n$. Table 5 (in conjunction with Tables 3 and 4) surely gives precisely those $n$ in the range $100<|n| \leq 200$ for which solutions to our representation problems exist.

Table 1

Solutions of $n=(x+y+z)^{3} / x y z$

$\begin{array}{rrl}n & h & \left\{\left(c_{0}, c_{1}, c_{2}\right),(u, u, w)\right\} \\ 2 & 0 & \{(1,1,2)(1,1,-1)\} \\ 7 & 1.06 & \{(1,1,7)(1,3,-1)\} \\ 10 & 1.84 & \{(5,2,1)(1,3,-7)\} \\ 12 & 0.62 & \{(1,4,3)(2,1,-2)\} \\ 14 & 1.70 & \{(1,1,14)(1,5,-1)\} \\ 15 & 0.79 & \{(1,3,5)(1,1,-1)\} \\ 19 & 5.16 & \{(1,1,19)(113,-198,13)\} \\ 22 & 3.59 & \{(1,2,1)(3,17,-41)\} \\ 27 * & - & \{(3,3,3)(1,1,1)\} \\ 31 & 2.29 & \{(1,1,31)(-13,3,1)\} \\ 32 & 0 & \{(1,1,32)(8,8,1)\} \\ 33 & 2.34 & \{(11,1,3)(2,13,5)\} \\ 36 & 0.19 & \{(1,4,9)(6,3,2)\} \\ 37 & 10.17 & \{(1,1,37)(31419,21622,2291)\} \\ 40 & 0.99 & \{(8,5,1)(1,2,6)\} \\ 41 & 5.89 & \{(1,1,41)(301,314,37)\} \\ 42 & 1.42 & \{(6,7,1)(1,1,5)\} \\ 43 & 4.64 & \{(1,1,43)(175,111,11)\} \\ 44 & 1.49 & \{(1,4,11)(2,3,-2)\} \\ 49 & 0.91 & \{(1,1,49)(14,21,1)\} \\ 50 & 0.44 & \{(1,2,25)(5,5,1)\} \\ 51 & 2.36 & \{(1,17,3)(5,1,-6)\} \\ 53 & 14.08 & \{(1,1,53)(226399,-1427644,81245)\} \\ 54 & 0 & \{(1,1,54)(9,9,1)\} \\ 56 & 1.16 & \{(2,2,14)(2,3,1)\} \\ 61 & 6.88 & \{(1,1,61)(4,971,-63)\} \\ 62 & 2.75 & \{(1,1,62)(9,17,1)\} \\ 65 & 4.66 & \{(1,1,65)(106,59,7)\} \\ 66 & 1.69 & \{(2,11,3)(2,1,3)\} \\ 67 & 22.03 & \{(1,1,67)(2774169542,-4286639393,70122339)\} \\ 68 & 2.21 & \{(4,17,1)(5,2,22)\} \\ 72 & 0.53 & \{(1,9,8)(6,2,3)\} \\ 73 & 26.87 & \{(1,1,73)(561473579210,293489655271,20805706971)\} \\ 75 & 0.27 & \{(1,25,3)(5,1,5)\} \\ & & \\ 12 & \end{array}$


Table 1 (continued)

\begin{tabular}{|c|c|c|}
\hline 76 & 1.54 & $\{(1,1,76)(6,10,-1)\}$ \\
\hline 78 & 5.89 & $\{(2,3,13)(241,89,65)\}$ \\
\hline 80 & 0.90 & $\{(1,2,40)(4,4,-1)\}$ \\
\hline 81 & 0.68 & $\{(1,1,81)(9,18,1)\}$ \\
\hline 82 & 10.24 & $\{(1,2,41)(15713,20721,1933)\}$ \\
\hline 83 & 19.31 & $\{(1,1,83)(93094675,-289590286,8561059)\}$ \\
\hline 85 & 7.04 & $\{(1,5,17)(-1311,307,44)\}$ \\
\hline 86 & 28.42 & $\{(2,43,1)(3,1,-19)\}$ and $\{(1,1,86)(17,33,1)\}$ \\
\hline 87 & 4.02 & $\{(1,3,29)(59,-35,1)\}$ \\
\hline 88 & 5.91 & $\{(2,2,22)(430,197,63)\}$ \\
\hline 89 & 14.83 & $\{(1,1,89)(1294733,-3329273,79972)\}$ \\
\hline 90 & 1.09 & $\{(9,5,2)(1,3,6)\}$ \\
\hline 91 & 2.38 & $\{(1,1,9])(9,17,1)\}$ \\
\hline 96 & 0.54 & $\{(1,32,3)(8,1,8)\}$ \\
\hline-1 & 0 & $\{(-1,1,1)(1,1,-1)\}$ \\
\hline-4 & 0.26 & $\{(-1,1,4)(2,-2,1)\}$ \\
\hline-6 & 0.67 & $\{(-1,2,3)(1,-1,1)\}$ \\
\hline-7 & 1.62 & $\{(-1,1,7)(4,5,-1)\}$ \\
\hline-9 & 0.15 & $\{(-1,1,9)(3,-3,1)\}$ \\
\hline-11 & 3.68 & $\{(-1,1,11)(12,43,-7)\}$ \\
\hline-13 & 1.86 & $\{(-1,1,13)(2,7,-1)\}$ \\
\hline-16 & 0.38 & $\{(-1,1,16)(4,-4,1)\}$ \\
\hline-18 & 0.48 & $\{(-1,9,2)(-3,1,3)\}$ \\
\hline-19 & 2.03 & $\{(-1,1,19)(1,8,-1)\}$ \\
\hline-20 & 1.23 & $\{(-1,5,4)(2,-2,3)\}$ \\
\hline-25 & 0.25 & $\{(-1,1,25)(5,-5,1)\}$ \\
\hline-29 & 6.85 & $\{(-1,1,29)(1084,453,-73)\}$ \\
\hline-30 & 0.99 & $\{(-2,3,5)(-1,1,1)\}$ \\
\hline-31 & 6.21 & $\{(-1,1,31)(503,445,-28)\}$ \\
\hline-33 & 1.65 & $\{(-3,11,1)(1,-1,7)\}$ \\
\hline-35 & 5.19 & $\{(-1,1,35)(173,172,-9)\}$ \\
\hline-36 & 0.46 & $\{(-1,1,36)(6,-6,1)\}$ \\
\hline-37 & 2.36 & $\{(-1,1,37)(1,-10,1)\}$ \\
\hline-38 & 7.11 & $\{(-1,1,38)(-1405,787,-63)\}$ \\
\hline-40 & 1.27 & $\{(-1,5,8)(1,-1,1)\}$ \\
\hline-42 & 3.13 & $\{(-1,6,7)(-13,5,7)\}$ \\
\hline-44 & 1.47 & $\{(-4,1,11)(-1,3,1)\}$ \\
\hline-45 & 0.97 & $\{(-9,1,5)(-1,6,3)\}$ \\
\hline-47 & 30.23 & $\{(-1,1,47)(-15725733900560,9548649243309,-457687455901)\}$ \\
\hline-48 & 0.25 & $\{(-1,2,24)(-4,4,1)\}$ \\
\hline-49 & 0.85 & $\{(-1,1,49)(7,-7,1)\}$ and $\{(-1,1,49)(-21,35,1)\}$ \\
\hline-52 & 3.08 & $\{(-1,1,52)(-34,50,1)\}$ \\
\hline$-5 \overline{5}$ & 4.19 & $\{(-1,5,11)(68,1,-13)\}$ \\
\hline-56 & 3.12 & $\{(-1,1,56)(-48,68,7)\}$ \\
\hline-57 & 4.39 & $\{(-1,1,57)(113,-65,3)\}$ \\
\hline-58 & 4.58 & $\{(-1,2,29)(103,53,-5)\}$ \\
\hline-59 & 12.11 & $\{(-1,1,59)(-85867,172479,11956)\}$ \\
\hline-61 & 12.35 & $\{(-1,1,61)(-140537,272152,8393)\}$ \\
\hline-63 & 1.55 & $\{(-1,1,63)(3,-12,1)\}$ \\
\hline-64 & 0.53 & $\{(-1,1,64)(8,-8,1)\}$ \\
\hline-65 & 14.94 & $\{(-1,5,13)(9,1,-1)\}$ and $\{(-1,1,65)(33,-73,7)\}$ \\
\hline-66 & 2.77 & $\{(-2,33,1)(5,-1,19)\}$ \\
\hline-67 & 20.62 & $\{(-1,1,67)(-607804759,401630884,64753875)\}$ \\
\hline-69 & 4.73 & $\{(-1,3,23)(-101,59,2)\}$ \\
\hline-70 & 8.28 & $\{(-2,5,7)(4,-1,1)\}$ and $\{(-1,1,70)(3,-13,1)\}$ \\
\hline-71 & 8.79 & $\{(-1,1,71)(2000,-9171,691)\}$ \\
\hline
\end{tabular}


Table 1 (continued)

\begin{tabular}{rrl}
\hline-73 & 3.05 & $\{(-1,1,73)(-13,29,1)\}$ \\
-75 & 1.21 & $\{(-25,3,1)(1,-5,20)\}$ \\
-76 & 3.54 & $\{-1,1,76)(-21,41,1)\}$ \\
-77 & 11.58 & $\{(-1,1,77)(-73081,126970,2439)\}$ \\
-79 & 11.12 & $\{(-1,1,79)(-13,4,1)\}$ \\
-80 & 2.30 & $\{(-2,40,1)(4,-3,44)\}$ \\
-81 & 0.38 & $\{(-1,1,81)(9,-9,1)\}$ \\
-84 & 5.84 & $\{(-3,4,7)(-2,1,2)\}$ and $\{(-3,4,7)(-1,2,1)\}$ \\
-91 & 7.95 & $\{(-1,1,91)(527,-2412,157)\}$ \\
-94 & 1.78 & $\{(-2,1,47)(3,-7,1)\}$ \\
-95 & 13.34 & $\{(-1,1,95)(-487427,164247,34628)\}$ \\
-96 & 0.86 & $\{(-8,3,4)(-1,4,4)\}$ \\
-97 & 56.69 & $\{(-1,1,97)(5,-14,1)\}$ and $\{(-1,1,97)(699,1435,-31)\}$ \\
-100 & 1.66 & $\{(-1,1,100)(10,-10,1)\}$ and $\{(-1,25,4)(-5,1,5)\}$ \\
\hline
\end{tabular}

Table 2

Solutions of $n=\left(a^{3}+b^{3}+c^{3}\right) / a b c$

\begin{tabular}{|c|c|c|}
\hline$n$ & $h$ & $(a, b, c)$ \\
\hline $3^{*}$ & - & $(1,1,1)$ \\
\hline 5 & 0 & $(1,1,2)$ \\
\hline 6 & 0.93 & $(1,2,3)$ \\
\hline 9 & 1.68 & $(2,3,7)$ \\
\hline 10 & 2.61 & $(5,7,18)$ \\
\hline 13 & 3.32 & $(9,13,38)$ \\
\hline 14 & 2.33 & $(2,7,13)$ \\
\hline 15 & 1.48 & $(7,-3,-1)$ \\
\hline 16 & 3.96 & $(70,-31,-9)$ \\
\hline 17 & 3.34 & $(5,18,37)$ \\
\hline 18 & 4.26 & $(13,42,95)$ \\
\hline 19 & 1.62 & $(1,5,9)$ \\
\hline 20 & 3.74 & $(61,-14,-13)$ \\
\hline 21 & 2.84 & $(2,13,21)$ \\
\hline 26 & 4.19 & $(9,38,91)$ \\
\hline 29 & 4.79 & $(27,43,182)$ \\
\hline 30 & 3.26 & $(2,21,31)$ \\
\hline 31 & 3.29 & $(37,-27,-1)$ \\
\hline 35 & 4.13 & $(97,-19,-14)$ \\
\hline 36 & 4.75 & $(151,-78,-7)$ \\
\hline 38 & 6.01 & $(70,151,629)$ \\
\hline 40 & 1.75 & $(9,-2,-1)$ \\
\hline 41 & 8.02 & $(1,2,9)$ and $(1,5,14)$ \\
\hline 44 & 6.53 & $(819,-554,-19)$ \\
\hline 47 & 6.41 & $(845,-367,-38)$ \\
\hline 51 & 3.51 & $(9,13,77)$ \\
\hline 53 & 2.45 & $(2,7,27)$ \\
\hline 54 & 3.26 & $(2,43,57)$ \\
\hline 57 & 4.76 & $(19,91,310)$ \\
\hline 62 & 15.91 & $(1950953,1513300,-13559153)$ \\
\hline 63 & 7.60 & $(247,903,-3775)$ \\
\hline 64 & 13.60 & $(119479,232736,-1338039)$ \\
\hline 66 & 1.51 & $(1,3,14)$ \\
\hline 67 & 9.13 & $(1133,7525,23517)$ \\
\hline 69 & 9.28 & $(2,57,73)$ and $(42,95,523)$ \\
\hline
\end{tabular}


Table 2 (continued)

\begin{tabular}{|c|c|c|}
\hline 70 & 13.98 & $(27083,896668,-1478979)$ \\
\hline 71 & 3.50 & $(67,-9,-7)$ \\
\hline 72 & 28.82 & $(-5450170263655,1012930784383,404512675962)$ \\
\hline 73 & 35.29 & $(89200900157319,2848691279889518,1391526622949983)$ \\
\hline 74 & 8.18 & $(133,2502,4607)$ \\
\hline 76 & 105.29 & $(2,-45,13)$ and $(1327,2196,-14911)$ \\
\hline 77 & 89.54 & $(5,-52,7)$ and $(67,630,1763)$ \\
\hline 83 & 3.22 & $(5,9,61)$ \\
\hline 84 & 3.08 & $(56,-31,-1)$ \\
\hline 86 & 4.41 & $(2,73,91)$ \\
\hline 87 & 2.38 & $(21,-5,-1)$ \\
\hline 92 & 30.96 & $(-35661385544981,548624531286,20446843218005)$ \\
\hline 94 & 157.05 & $(19,746,945)$ and $(27,182,673)$ \\
\hline 96 & 3.07 & $(38,-5,-3)$ \\
\hline 98 & 17.41 & $(14154192,2559169,-59978401)$ \\
\hline 99 & 22.83 & $(1832602198,-14466072543,1150522313)$ \\
\hline-4 & 0.53 & $(1,-1,2)$ \\
\hline-9 & 0.65 & $(1,-1,3)$ \\
\hline-10 & 1.73 & $(4,-7,-1)$ \\
\hline-11 & 2.69 & $(4,-19,-9)$ \\
\hline-12 & 2.81 & $(3,-19,-14)$ \\
\hline-16 & 8.66 & $(1,-1,4)$ and $(2,-5,13)$ \\
\hline-17 & 1.91 & $(1,-9,-7)$ \\
\hline-21 & 4.07 & $(37,-78,-7)$ \\
\hline-22 & 1.50 & $(1,-9,-4)$ \\
\hline-24 & 1.02 & $(2,-7,-1)$ \\
\hline-25 & 6.33 & $(1,-1,5)$ and $(1,-7,-2)$ \\
\hline-27 & 5.30 & $(28,-279,-109)$ \\
\hline-28 & 7.09 & $(325,-1813,-362)$ \\
\hline-29 & 4.62 & $(9,-127,-74)$ \\
\hline-32 & 13.42 & $(401791,927041,-72252)$ \\
\hline-33 & 3.03 & $(3,-35,-13)$ \\
\hline-34 & 3.00 & $(7,-31,-4)$ \\
\hline-35 & 9.84 & $(14220,23233,-1333)$ \\
\hline-36 & 1.34 & $(1,-1,6)$ \\
\hline-37 & 4.84 & $(52,-193,-19)$ \\
\hline-38 & 14.38 & $(28251,1934524,-1581475)$ \\
\hline-40 & 8.17 & $(2692,4345,-217)$ \\
\hline-44 & 5.04 & $(19,-234,-67)$ \\
\hline-45 & 4.96 & $(52,-223,-21)$ \\
\hline-46 & 3.29 & $(8,-43,-5)$ \\
\hline-47 & 4.94 & $(9,-221,-196)$ \\
\hline-48 & 17.35 & $(28843468,39825737,-1580745)$ \\
\hline-49 & 11.02 & $(1,-1,7)$ and $(1,-18,-7)$ \\
\hline-50 & 16.56 & $(1980727,24383561,-5920704)$ \\
\hline-53 & 22.38 & $(-228354001,4235900895,6254246146)$ \\
\hline-55 & 4.78 & $(7,-163,-76)$ \\
\hline-56 & 20.37 & $(329267696,1064663271,-63278951)$ \\
\hline-57 & 1.28 & $(1,-13,-3)$ \\
\hline-59 & 9.39 & $(817,17739,-6244)$ \\
\hline-60 & 47.87 & $(2,19,-3)$ and $(5,-117,-158)$ \\
\hline-63 & 26.03 & $(-77354606721,319899185941,20707834820)$ \\
\hline-64 & 1.56 & $(1,-1,8)$ \\
\hline-65 & 15.54 & $(4094597,9326853,-354485)$ \\
\hline-66 & 8.27 & $(3423,4432,-127)$ \\
\hline-67 & 13.67 & $(93733,1399411,-308584)$ \\
\hline
\end{tabular}


Table 2 (continued)

\begin{tabular}{rrll}
\hline-68 & 6.99 & $(35,-1251,-914)$ \\
-72 & 35.41 & $(1,26,-9)$ and $(6,91,-19)$ \\
-73 & 9.68 & $(13483,24577,-715)$ \\
-76 & 3.75 & $(10,-73,-7)$ \\
-77 & 8.93 & $(853,12581,-2394)$ \\
-79 & 7.16 & $(823,-1764,-43)$ \\
-80 & 27.07 & $(-134711653167,88915731292,979804259375)$ \\
-81 & 1.47 & $(1,-1,9)$ \\
-82 & 11.16 & $(-1736,67295,73841)$ \\
-84 & 6.28 & $(28,-793,-279)$ \\
-85 & 9.23 & $(307,-12857,-7690)$ \\
-86 & 42.69 & $(5786669814541025089,319409653453108344,-1208131767609148369)$ \\
-87 & 21.70 & $(-64989513,2989660076,2310135877)$ \\
-88 & 134.44 & $(4,-79,175)$ and $(-27,1147,1220)$ \\
-89 & 4.53 & $(13,-189,-31)$ \\
-90 & 2.91 & $(1,-28,-9)$ \\
-92 & 170.88 & $(9,-208,439)$ and $(38,-1629,2701)$ \\
-94 & 3.95 & $(11,-91,-8)$ \\
-95 & 57.73 & $(1,-13,36)$ and $(11,-387,-152)$ \\
-100 & 1.73 & $(1,-1,10)$ \\
\hline
\end{tabular}

Table 3

Pairs of solutions of $n=(x+y+z)^{3} / x y z$ when rank is 2

\begin{tabular}{lll}
\hline 104 & $\{(1,8,13),(1,1,-1)\}$ & $\{(13,8,1),(2,3,22)\}$ \\
126 & $\{(1,6,21),(3,1,-1)\}$ & $\{(21,1,6),(1,9,5)\}$ \\
150 & $\{(1,25,6),(5,1,-5)\}$ & $\{(2,75,1),(10,1,35)\}$ \\
157 & $\{(1,1,157),(-124,75,1)\}$ & $\{(1,1,157),(47247,18175,1022)\}$ \\
165 & $\{(3,5,11),(1,2,1)\}$ & $\{(1,5,33),(188,-137,13)\}$ \\
172 & $\{(1,4,43),(3,-7,1)\}$ & $\{(1,1,172),(198,10,-7)\}$ \\
199 & $\{(1,1,199),(-81,29,1)\}$ & $\{(1,1,199),(-21193,508743,-13718)\}$ \\
& & \\
-100 & $\{(-1,1,100),(10,-10,1)\}$ & $\{(-1,25,4),(-5,1,5)\}$ \\
-154 & $\{(-1,7,22),(3,-1,1)\}$ & $\{(-1,1,154),(37,73,-1)\}$ \\
-163 & $\{(-1,1,163),(-17,8,1)\}$ & $\{(-1,1,163),(79880,156389,-2021)\}$ \\
-169 & $\{(-1,1,169),(13,-13,1)\}$ & $\{(-1,1,169),(-13,39,1)\}$ \\
-180 & $\{(-1,36,5),(-6,1,6)\}$ & $\{(-1,4,45),(3,-3,1)\}$ \\
-188 & $\{(-1,4,47),(33,16,-1)\}$ & $\{(-1,1,188),(10521,26515,-349)\}$ \\
\hline
\end{tabular}


Table 4

Pairs of solutions of $n=\left(a^{3}+b^{3}+c^{3}\right) / a b c$ when rank is 2

\begin{tabular}{rll}
\hline 105 & $(2,91,111)$ & $(35,1171,1854)$ \\
106 & $(1,35,54)$ & $(1342,15929,46683)$ \\
126 & $(2,111,133)$ & $(843543,6610037,26297374)$ \\
129 & $(31,774,1679)$ & $(39,-619,76)$ \\
136 & $(45,-1118,203)$ & $(126,2797,-7141)$ \\
149 & $(1,14,45)$ & $(2,133,157)$ \\
151 & $(9,-133,13)$ & $(9,-1054,637)$ \\
154 & $(2,13,63)$ & $(62,1183,3285)$ \\
160 & $(43,-3691,1764)$ & $(182,-4559,711)$ \\
161 & $(7,-95,8)$ & $(11,38,259)$ \\
174 & $(5,7,78)$ & $(2,157,183)$ \\
186 & $(5,-252,67)$ & $(2269,15938,81711)$ \\
195 & $(7,15,143)$ & $(39,2279,703)$ \\
196 & $(18,19,-259)$ & $(4221,378995,-632186)$ \\
& & $(1634,19431,-1991)$ \\
-116 & $(74,3951,-1679)$ & $(670,-12309,-1891)$ \\
-120 & $(47295,-97256,743681)$ & $(21,-341,-43)$ \\
-129 & $(1,-63,-37)$ & $(661,-8965,-936)$ \\
-130 & $(16,-533,-139)$ & $(248,-9581,-2811)$ \\
-135 & $(-172,-6669,1873)$ & $(132842,-14578235,20541483)$ \\
-140 & $(1,-49,-18)$ & $(143,-938,4491)$ \\
-149 & $(117,-3181,-584)$ & $(335,-13394,-3591)$ \\
-152 & $(103,-1764,-199)$ & $(-2993,112329,26702)$ \\
-160 & $(14,-157,-11)$ & $(976,-4111,26847)$ \\
-179 & $(36,-2521,-3395)$ & $(-238975823,-23863710,13306223)$ \\
-180 & $(21,-1333,-494)$ & $(19,-889,-234)$ \\
-181 & $(7,-302,-73)$ & $(1,-481,545)$ \\
-193 & $(1,-31,-5)$ & $(539,-14643,-38168)$ \\
-195 & $(13,-1204,1983)$ & $(7540511,-7518559,-105413546)$ \\
-196 & $(1,-1,14)$ & \\
\hline & &
\end{tabular}


ANDREW BREMNER AND RICHARD K. GUY

Table 5

Values of $n(n>0)$ such that (1) has rank 1:

\begin{tabular}{|c|c|c|c|c|c|c|c|c|c|c|c|}
\hline 101 & 109 & 111 & 113 & 114 & 115 & 116 & 118 & 122 & 123 & 124 & $(125)$ \\
\hline 127 & 131 & 132 & 133 & 136 & 138 & 140 & 142 & 145 & 146 & 149 & 152 \\
\hline 153 & 159 & 160 & 161 & 163 & 164 & 167 & 169 & 170 & 173 & 174 & 175 \\
\hline 176 & 177 & 179 & 180 & 182 & 183 & 184 & 185 & 186 & 187 & 189 & 193 \\
\hline 196 & 198 & & & & & & & & & & \\
\hline \multicolumn{12}{|c|}{ Values of $m(n=-m, m>0)$ such that (1) has rank 1 : } \\
\hline 102 & 104 & 105 & 106 & 107 & 109 & 112 & 113 & 114 & 115 & 117 & 118 \\
\hline 119 & 121 & 123 & 124 & 126 & 128 & 129 & 131 & 133 & 134 & 136 & 139 \\
\hline 140 & 144 & 148 & 151 & 152 & 153 & 157 & 158 & 160 & 162 & 164 & 167 \\
\hline 168 & 171 & 172 & 174 & 177 & 178 & 179 & 182 & 184 & 186 & 187 & 189 \\
\hline 190 & 191 & 192 & 193 & 194 & 196 & 197 & 198 & 199 & & & \\
\hline \multicolumn{12}{|c|}{ Values of $n(n>0)$ such that (3) has rank 1: } \\
\hline 101 & 102 & 103 & 107 & 108 & 109 & 110 & 112 & 113 & 116 & 117 & 119 \\
\hline 120 & 122 & 123 & 124 & 127 & 128 & 130 & 132 & 133 & 142 & 143 & 145 \\
\hline 147 & 148 & 155 & 156 & 158 & 159 & 162 & 164 & 166 & 167 & 172 & 175 \\
\hline 177 & 178 & 181 & 185 & 187 & 189 & 190 & 191 & 192 & 197 & & \\
\hline \multicolumn{12}{|c|}{ Values of $m(n=-m, m>0)$ such that (3) has rank 1: } \\
\hline 101 & 103 & 105 & 108 & 111 & 112 & 113 & 114 & 117 & 118 & 121 & 122 \\
\hline 123 & 124 & 128 & 132 & 136 & 137 & 143 & 144 & 145 & 150 & 155 & 157 \\
\hline 158 & 159 & 163 & 164 & 166 & 168 & 169 & 170 & 172 & 174 & 177 & 178 \\
\hline 182 & 183 & 184 & 186 & 188 & 189 & 192 & 194 & 198 & 199 & 200 & \\
\hline
\end{tabular}




\section{REFERENCES}

1. C. Batut, D. Bernardi, H. Cohen and M. Oliver, Pari/GP, Version 1.39.

2. ANDrew Bremner, Richard K. GuY and Richard J. Nowakowski, Which integers are representable as the product of the sum of three integers with the sum of their reciprocals? Math. Comput. 61 (1993) (Lehmer Memorial issue), 117-130; MR 93k: 11021.

3. J. W. S. CAssels, On a Diophantine equation, Acta Arith. 6 (1960), 47-52.

4. J. W. S. Cassels and G. Sansone, Sur le problème de M. Werner Mnich, Acta Arith. 7 (1962), 187-190.

5. MAURICE Craig, Integer Values of $\sum\left(x^{2} / y z\right), J$. Number Theory 10 (1978), 62-63.

6. ERIK DoFs, On some classes of homogeneous ternary cubic Diophantine equations, Ark. Mat. 13 (1975), 29-72.

7. ERIK Dofs, Solutions of $x^{3}+y^{3}+z^{3}=n x y z$, Acta Arith., to appear.

8. Richard K. Guy, Problem 10382, Amer. Math. Monthly 101 (1994), 473; pre-publication solutions by Arthur Baragar and Brian Peterson.

9. Richard K. GuY, Problem $93: 31$, Western Number Theory Problems, $93: 12: 21$.

10. Sharon A. Kineke, Integers representable by $(x+y+z)^{3} / x y z$, Internat. J. Math. Sci., submitted.

11. Joseph Konhauser, Dan Velleman and Stan Wagon, Which Way Did the Bicycle Go?, Dolciani Series, Math. Assoc. Amer., 1996, Problem 83 (Problem 477 from the Joseph Konhauser collection), "A sum of fractions." Some solutions by Underwood Dudley.

12. S. P. MohantY, A system of cubic Diophantine equations, J. Number Theory 9 (1977), 153-159.

13. W. SIER PIŃSKI, Remarques sur le travail de $M$. J. W. S. Cassels, "On a Diophantine Equation", Acta Arith. 6 (1960), 469-471.

14. W. Sier PIŃski, 250 Problems in Number Theory (Elsevier, New York, 1970), 78-81.

15. E. Thomas and A. T. VASQuez, Diophantine equations arising from cubic number fields, J. Number Theory 13 (1981), 398-414.

16. ANDrew WiLes, Modular elliptic curves and Fermat's Last Theorem, Ann. of Math. (2) 141 (1995), 443-551.

Department of Mathematics

ARIZONA STATE UNIVERSITY

TEMPE AZ 85287-1804

U.S.A.

E-mail address: andrew@math.la.asu.edu
Department of Mathematics and Statistics

THE UNIVERSITY OF CALGARY

Calgary, Alberta

CANADA T2N 1N4

E-mail address: rkg@cpsc.ucalgary.ca 